\title{
Structuration profonde dans la région de Bir M'Chergua (Nord-Est de la Tunisie). Implications hydrogéologiques
}

\section{Deep structure in the Bir M'Chergua region (North-East of Tunisia). Hydrogeological implications}

\author{
A. Hamed Ferjani ${ }^{1,2}$, R. Guellala ${ }^{1,2}$, A. Amiri ${ }^{1}$, A. Merzougui ${ }^{3}$, M.H. Inoubli ${ }^{1}$ \\ ${ }^{1}$ Unité de Recherche de Géophysique Appliquée aux Matériaux et Minerais, Département de Géologie, Faculté des Sciences \\ de Tunis, Campus Universitaire, 1060 Tunis, Tunisie. Email: ferjaniasma2013@gmail.com, rihabguellala@gmail.com; \\ ORCID ID: https://orcid.org/0000-0001-8307-9794, https://orcid.org/0000-0002-4680-291X, https://orcid.org/0000-0002- \\ 0972-8319, https://orcid.org/0000-0002- 9329-2780 \\ ${ }^{2}$ Laboratoire de Géoressources, CERTE, Technopôle de Borj Cedria, Tunisie \\ ${ }^{3}$ Commissariat Régional de Développement Agricole de Zaghouan, Tunisie. ORCID ID: https://orcid.org/0000-0002- 1435-1261
}

\section{RÉSUMÉ}

La région de Bir M'Chergua sise à cinquante kilomètres de la capitale Tunis constitue un pôle économique important en Tunisie. Cependant la pénurie en eau résultante de la succession de nombreuses périodes de sécheresse a généré une notable dégradation de diverses activités industrielles, touristiques et agricoles dans cette région. L'exploitation des eaux souterraines peut pallier à cette situation mais elle nécessite une meilleure caractérisation des aquifères. Dans ce contexte, est réalisée la présente étude qui vise à apporter des précisions au schéma hydrogéologique de la région de Bir M'Chergua en déterminant la structuration profonde à partir de l'interprétation des données gravimétriques.

Des cartes d'anomalie résiduelle, de la dérivée verticale et du gradient horizontal total sont calculées à partir de l'anomalie de Bouguer pour identifier et délimiter les sources perturbatrices. La technique de déconvolution d'Euler est aussi appliquée pour estimer la profondeur de ces sources.

La carte structurale issue de l'interprétation gravimétrique permet de mettre en évidence des structures enfouies et des discontinuités de différentes directions et profondeurs qui peuvent influencer énormément la géométrie et le fonctionnement du système aquifère de Bir M'Chergua. En effet, la mise en évidence des accidents tectoniques profonds de plus de $1500 \mathrm{~m}$ aux alentours du Dôme du Jebel Oust permet d'expliquer l'origine et la forte salinité des eaux thermales dans ce secteur; les accidents ont favorisé une pénétration profonde des eaux de recharge ainsi que leur contact avec les terrains triasiques.

Mots clés: Tunisie; Bir M'cherga; structures profondes; schéma hydrogéologique; gravimétrie.

\section{ABSTRACT}

The Bir M'Chergua region, located fifty kilometers from the capital Tunis, is an important economic pole in Tunisia. However, the water shortage resulting from many successive drought periods has led to a significant degradation of the various industrial, tourist and agricultural activities in this region. Groundwater exploitation can alleviate this situation, but it requires a better characterization of aquifers. In this context, the present study aims to

Recibido el 8 de Agosto de 2017 / Aceptado el 21 de febrero de 2018 / Publicado online el 25 de abril de 2018

Citation / Cómo citar este artículo: Hamed Ferjani, A. et al., (2018). Structuration profonde dans la région de Bir M'Chergua (Nord-Est de la Tunisie). Implications hydrogéologiques. Estudios Geológicos 74(1): e075. https://doi.org/10.3989/egeol.42955.461.

Copyright: () 2018 CSIC. This is an open-access article distributed under the terms of the Creative Commons Attribution-Non 4.0 International License. 
provide precisions on the Bir M'Chergua hydrogeological scheme by determining the subsurface structures from gravimetric data interpretation.

Residual anomaly, vertical derivative and total horizontal gradient maps are calculated from the Bouguer anomaly to identify and delimit the causative sources. The Euler deconvolution technique is also applied to estimate these sources depth.

The structural map resulting from gravimetric interpretation highlights buried structures and discontinuities of different direction and depth that can greatly influence the Bir M'Chergua aquifer system geometry and functioning. In fact, the highlighting of tectonic features at more than $1500 \mathrm{~m}$ depth around the Jebel Oust dome explains the origin and the high salinity of the thermal waters in this sector; such tectonic accidents have favored a deep penetration of the recharge water as well as their contact with the Triassic terrains.

Keywords: Tunisia; Bir M'Chergua; deep structures; hydrogeological scheme; gravimetry.

\section{Introduction}

En Tunisie, l'importante pénurie en eau due à plusieurs années de sécheresse a touché énormément les activités économiques et domestiques; elle a été à l'origine des fréquentes coupures en eau enregistrées dans tout le pays. Ainsi, l'exploitation des eaux souterraines est devenue une nécessité pour combler le besoin en eau. Cependant, la réussite de cette stratégie demande une bonne compréhension de la structuration profonde et de son effet sur les caractéristiques des aquifères afin d'assurer le succès des forages à réaliser. Dans ce contexte, est réalisé le présent travail qui intéresse la région de Bir M'Chergua située au nord-est de la Tunisie (Fig. 1) à cinquante kilomètres de la capitale et qui constitue un important pôle économique comportant différentes activités industrielles, agricoles et touristiques. Par ailleurs, Bir M'Cherga est caractérisée par la station thermale du Jebel Oust connue mondialement pour ses soins thérapeutiques.

Ce travail mené en collaboration entre l'Unité de Recherches de Géophysique Appliquée aux Matériaux et Minerais, le Centre de Recherches et de Technologies des Eaux, le Commissariat Régional de Développement Agricole de Zaghouan et l'Office National des Mines, vise à établir une carte structurale dans la région de Bir M'Chergua qui servira comme un document de base qui permet d'orienter les programmes d'exploitation des aquifères dans cette région. Cependant, les traits tectoniques profonds responsables de la structuration d'un aquifère ne peuvent pas être déterminés seulement par les données de forages; c'est pour cette raison que nous avons eu recours à la méthode gravimétrique appliquée avec succès dans différentes études hydrogéologiques (Najine et al., 2006; El Goumi et al., 2010; Ouerghi et al., 2013).

\section{Contexte géologique}

La région de Bir M'Chergua constitue un point de convergence des principales zones structurales de la Tunisie: la plateforme pélagienne (Sahel), la partie nord de l'Atlas (Atlas septentrional), l'Axe $\mathrm{N}-\mathrm{S}$ et la faille de Zaghouan (Fig. 1a). Cette région est caractérisée par différents traits tectoniques (Fig. 1b); les plus importantes sont les anticlinaux de Jebel Oust, Jebel Aziz et Jebel Ez Zebbas, les synclinaux de Henchir el Morra-Jebel Es Sba, de Bled el Mengoub et de Khelidia -Ain el Djour et le faisceau de failles transverses de direction NW-SE qui tronque au Sud le dôme du Jebel Oust tout en mettant en contact les dépôts crétacés inférieurs et ceux d'âge Eocène (Fig. 2).

$\mathrm{Du}$ point de vue stratigraphique, les affleurements géologiques dans le secteur d'étude s'étendent du Trias au Quaternaire (Fig. 2a). Les dépôts triasiques caractérisant Jebel Aziz et Jebel Zebbas sont représentés par du gypse, des grès, des calcaires et de dolomies (Fig. 2b). La série jurassique identifiée dans les massifs d'Oust et Aziz est composée de calcaires avec des intercalations marneuses (Fig. 2b). Les dépôts liasiques constituent le cœur du dôme du Jebel Oust. Le Crétacé est marqué par des alternances marno-calcaires (Fig. 2b) qui couvrent une large partie de la feuille de Bir M'Chergua (Fig. 2a). L'Eocène est représenté par des calcaires intercalés d'argiles riches en gypse (Fig. 2b). La série oligocène épaisse de 275 m (Jauzein, 1957) est formée de grès, sables et argiles. Les sédiments mio-pliocènes sont composés de calcaires, argiles sableuses, grès et conglomérats. Le Quaternaire est caractérisé par une croûte calcaire, d'argiles à intercalations sableuses, des galets et d'alluvions éoliennes argilogypseuses (Fig. 2b). 


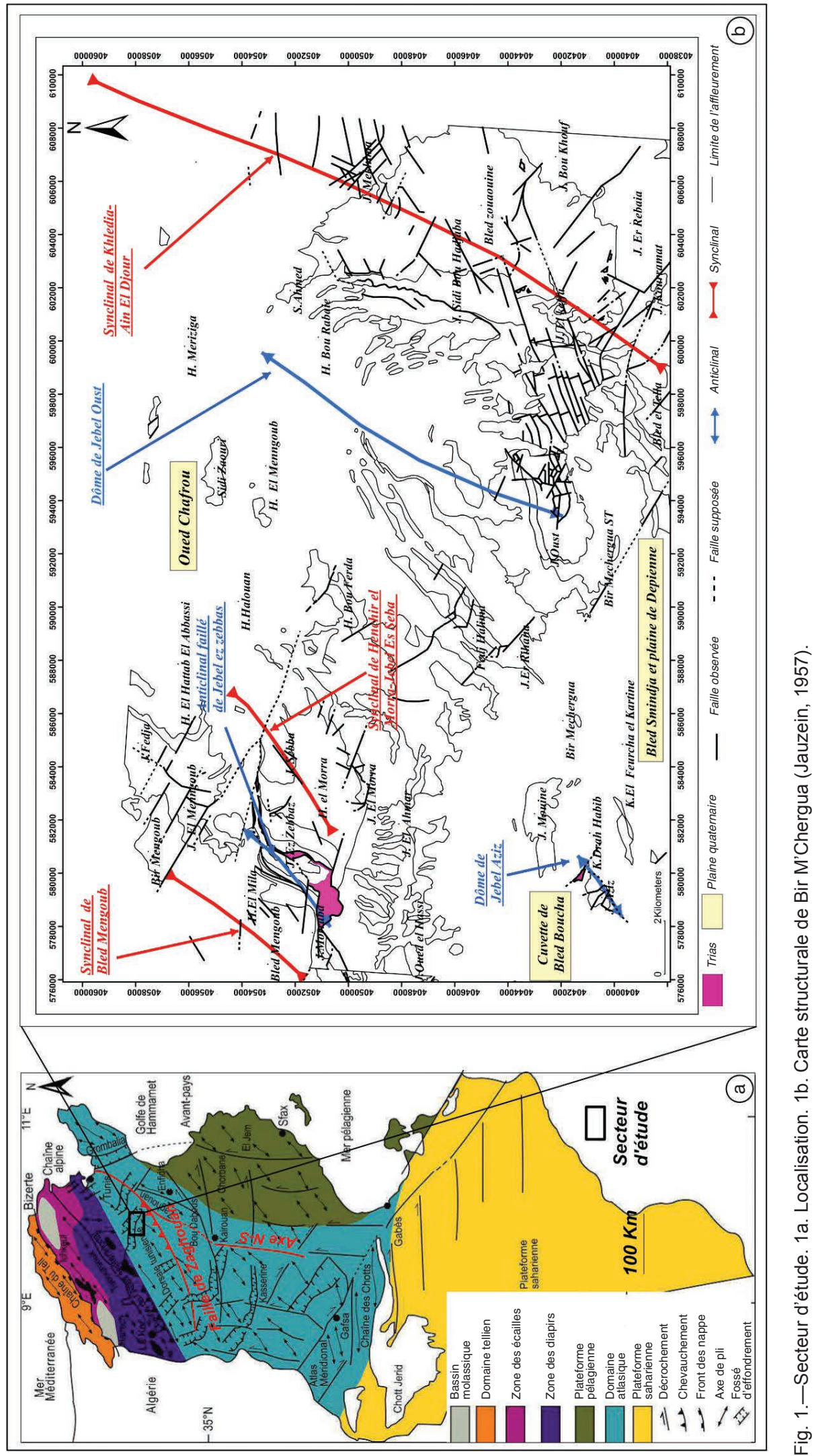




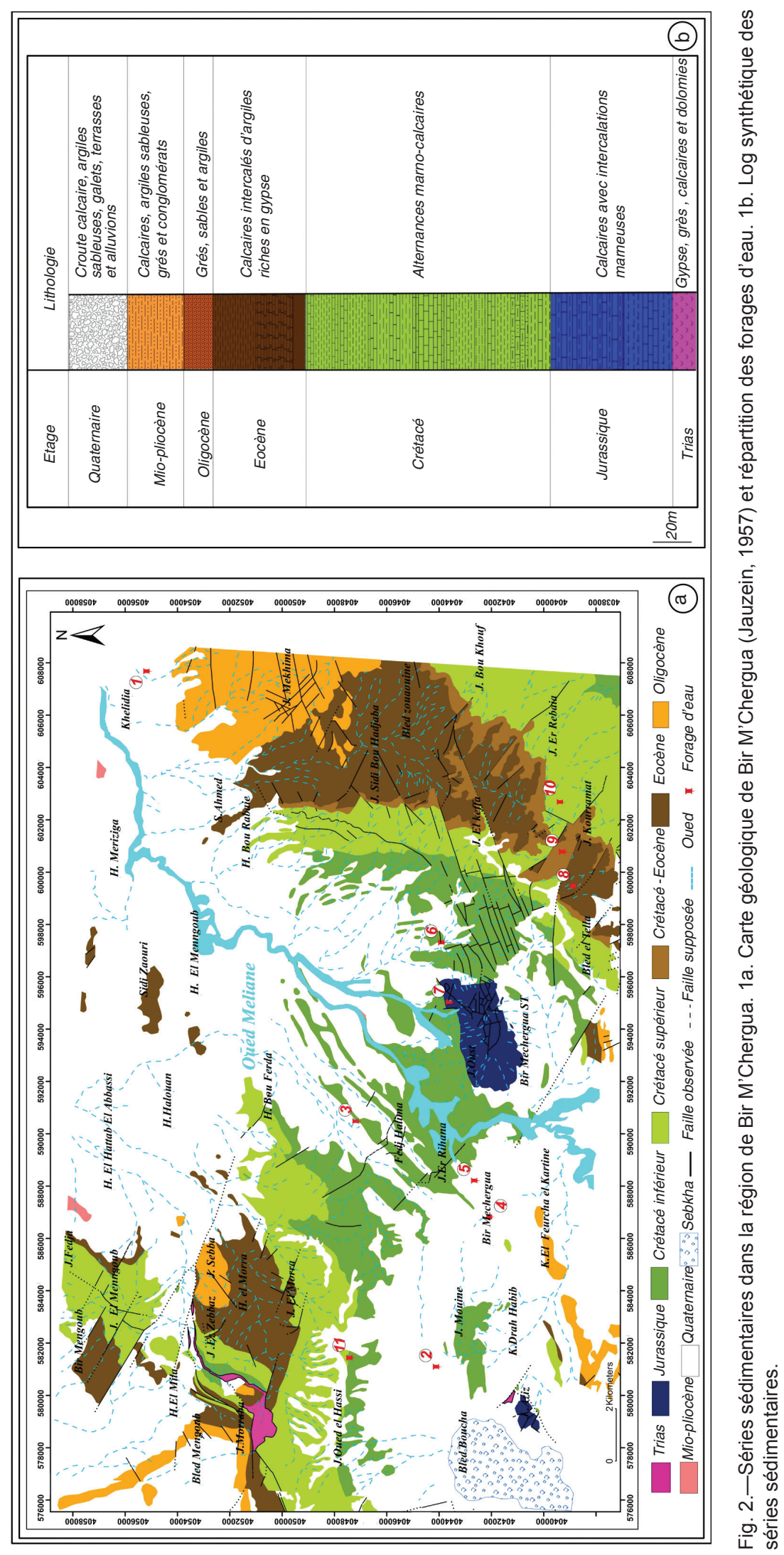




\section{Contexte hydrogéologique}

Les données de forages d'eau relatives à la région de Bir M'Chergua mises à notre disposition par le Commissariat Régional de Développement Agricole de Zaghouan montrent que cette région est caractérisée par différents aquifères dont l'âge s'étend du Jurassique au Quaternaire. Onze forages représentatifs sont sélectionnés et mentionnés sur la carte géologique (Fig. 2a).

$\mathrm{Au}$ niveau du Jebel Oust, les calcaires jurassiques à la base du forage 7 montrent des résistivités allant de 100 à $200 \mathrm{Ohmm}$ (Fig. 3). Cependant, les eaux jaillissant de ce forage (Fig. 2a) sont caractérisées par une température de l'ordre de $50^{\circ} \mathrm{C}$ et une salinité élevée de $16 \mathrm{~g} / 1$. Cette contradiction impose une question sur l'origine des eaux thermales du Jebel Oust.

Les dépôts crétacés qui affleurent largement dans la région de Bir M'Chergua renferment plusieurs aquifères composés de calcaires fissurés à différente perméabilité. Ceux d'âge barrémien et campanien -maestrichtien présentent de meilleures caractéristiques (Table 1) et sont ainsi les plus sollicités.

Les calcaires éocènes constituent un important aquifère particulièrement exploité dans la partie sud-est de la région de Bir M'Chergua. Etant donné la grande variabilité du taux de fracturation de ces calcaires, différents résultats sont obtenus au niveau des forages qui les traversent; le forage 9 (Fig. 1b) est négatif alors que le forage 8 situé à proximité (Fig. 1b) est productif avec un débit de 8,57 1/s et une salinité de l'eau de $1,66 \mathrm{~g} / 1$. Dans ce forage, les calcaires éocènes sont caractérisés par une transmissivité de $0,8110^{-4} \mathrm{~m}^{2} / \mathrm{s}$ et des valeurs de résistivité déterminées à partir des enregistrements diagraphiques qui atteignent $200 \mathrm{Ohm} m$ (Fig. 4).

Dans la zone nord-est de Bir M'Chergua, ce sont plutôt les aquifères oligocène et quaternaire qui sont captés. Au niveau du forage 1 (Fig. 1b), l'aquifère oligocène composé essentiellement de sables et de grès est caractérisé par une bonne qualité de l'eau (salinité de l'eau: $0,8 \mathrm{~g} / \mathrm{l}$ ) et un débit relativement fort de 18 1/s. Peu profond, l'aquifère alluvial quaternaire est exploité par des puits de surface. Il s'étend vers l'est à la plaine de Mornag.

\section{Données et méthodologie}

650 mesures gravimétriques couvrent le secteur d'étude selon une maille presque régulière d'un point par $\mathrm{km}^{2}$. Elles sont fournies par 1'Office National des Mines et acquises en 2009 avec un gravimètre Scintrex CG3 capable d'offrir une précision de $10^{-3}$ mGals (Gabalda et al., 2003).

Le positionnement des stations gravimétriques est effectué par un système GPS bi-fréquentiels de type Leica. La projection adoptée est Lambert Nord en utilisant l'ellipsoïde de Clark 1880 et le datum Carthage.

L'anomalie de Bouguer a été calculée en tenant compte de toutes les corrections (correction à l'air libre, correction de Bouguer) et en adoptant une densité de réduction de $2,36 \mathrm{~g} / \mathrm{cm}^{3}$ déterminée par de mesures directes sur des échantillons obtenus des affleurements des formations géologiques représentatives de la zone d'étude et de mesures indirectes par la méthode de Nettleton et celle de triplets (Schoeffler, 1975; Nely, 1986).

Afin d'exploiter au mieux les mesures gravimétriques et en extraire le maximum d'informations sur l'organisation du sous-sol de la région de Bir M'Chergua, différentes techniques qui ont généré différentes cartes ont été appliquées en utilisant le logiciel Geosoft Oasis Montaj 6.4. Geosoft est une société de développement des logiciels géophysiques et géologiques basée à Toronto, Canada et fondée en 1982 pa Ian MacLeod et Colin Reeves.

Les cartes d'anomalie de Bouguer, d'anomalie résiduelle, de la dérivée verticale et du gradient horizontal sont calculées.

La carte d'anomalie de Bouguer reflète toutes les variations de la densité en profondeur (Schoeffler, 1975; Blakely, 1996). Vu que notre objectif est l'étude des aquifères, nous allons nous focaliser essentiellement sur les hétérogénéités de masse proches de la surface.

La technique de prolongement vers le haut (Jacobsen, 1987; Zeng et al., 2007; Jacoby \& Smilde, 2009) permet de filtrer les structures de faibles longueur d'ondes proches de la surface laissant apparaître les structures de plus grandes longueurs d'ondes (c'est un filtre passe bas); l'anomalie régionale est engendrée par des sources 


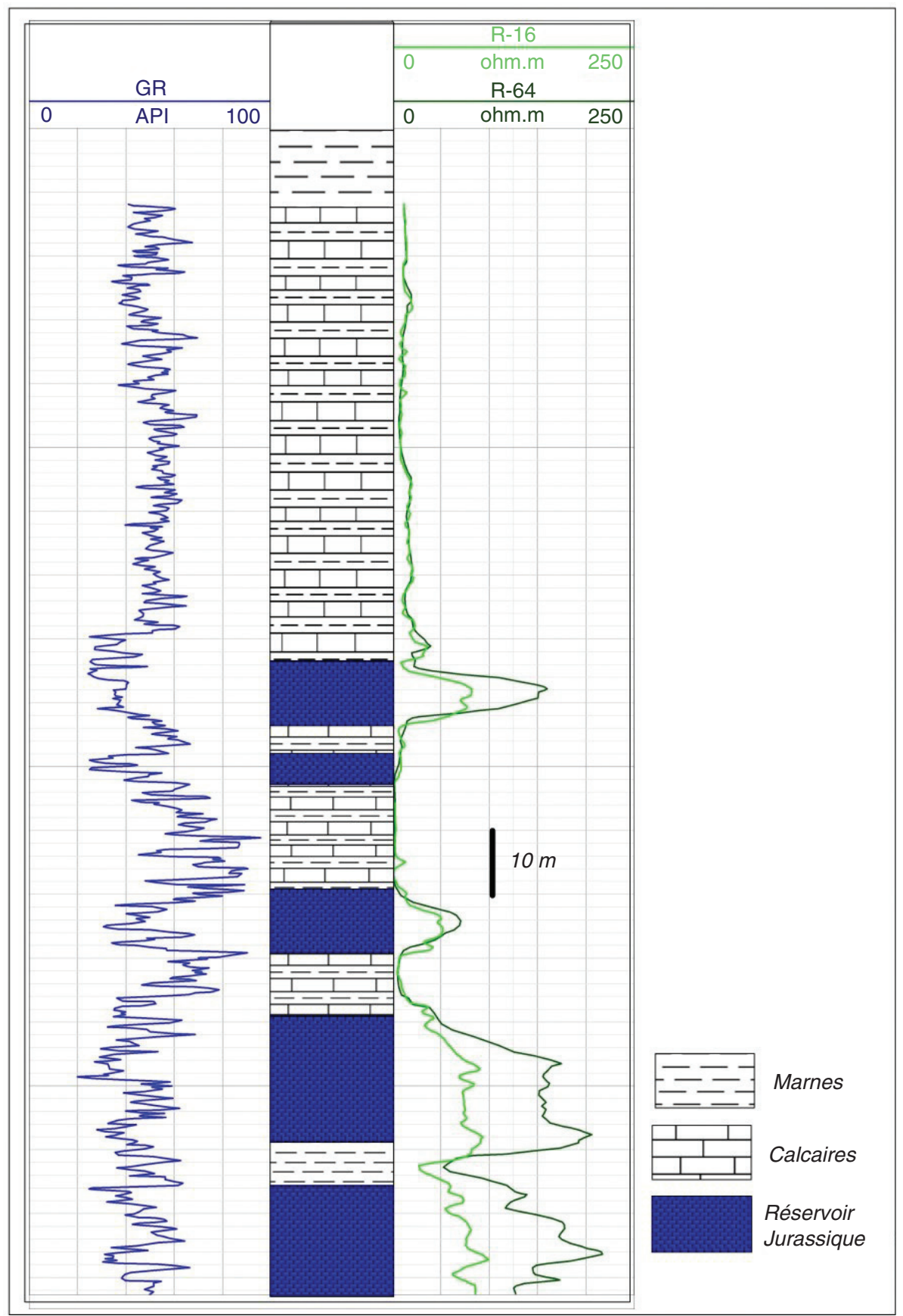

Fig. 3.-Log lithologique et enregistrements diagraphiques dans le forage 7.

Table 1.- Caractéristiques des aquifères crétacés.

\begin{tabular}{lccc}
\hline Aquifère crétacé & Numéro du forage sélectionné & Débit de l'eau (l/s) & Salinité de l'eau (g/l) \\
\hline Valanginien-Hauterivien & 6 & 2,3 & 3,7 \\
Barrémien & 3 & 9 & 2 \\
Vraconien & 11 & 2,8 & 3,5 \\
Campanien -Maestrichtien & 10 & 4 & 1,1 \\
\hline
\end{tabular}




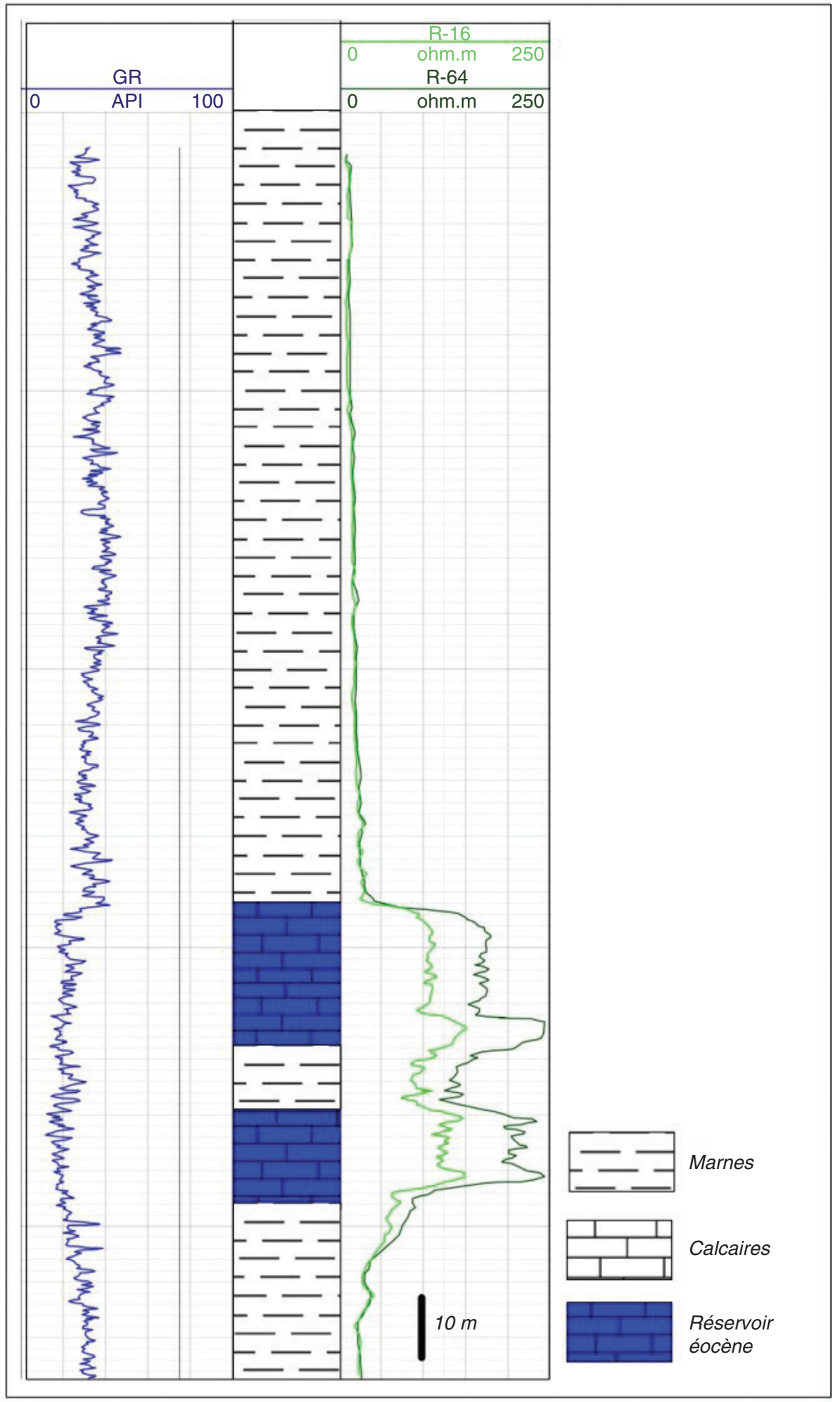

Fig. 4.-Log lithologique et enregistrements diagraphiques dans le forage 8. 
profondes caractérisées par une large extension horizontale alors que l'anomalie résiduelle résulte de sources situées près de la surface.

La dérivée verticale (Blakely, 1996) permet d'accentuer les anomalies gravimétriques de courtes longueurs d'ondes et de renforcer l'amplitude des structures géologiques proches de la surface. Cette technique tend à réduire le phénomène de coalescence des anomalies (Dubois et al., 2011).

La technique du gradient horizontal est très utilisée pour localiser les discontinuités en subsurface à partir de données gravimétriques (Fedi \& Florio, 2001). Les maxima du gradient horizontal coïncident aux positions de ces discontinuités (Dubois et al., 2011).

La technique de déconvolution d'Euler, appliquée aux cartes magnétiques (Thompson, 1982) et gravimétriques (Reid et al., 2003) permet d'estimer la localisation des sources d'anomalies ainsi que leur profondeur.

Les solutions de la déconvolution d'Euler dépendent des paramètres suivants: l'indice structural (IS), la fenêtre $(\mathrm{F})$ et la tolérance $(\mathrm{T})$.

Dans notre étude, nous avons retenu les valeurs correspondant à IS $=0, \mathrm{~F}=8 \mathrm{~km}$ et $\mathrm{T}=20 \%$ qui ont donné le meilleur regroupement des solutions. Par ailleurs, IS $=0$ est généralement utilisé pour identifier les failles (Reid et al., 2003)

\section{Résultats et discussions}

\section{Carte de l'anomalie de Bouguer}

La carte de l'anomalie de Bouguer (Fig. 5) ne montre que des valeurs positives allant de 0 à $25 \mathrm{mGal}$ malgré l'abondance des terrains quaternaires à faible densité dans la région de Bir M'Chergua (Fig. 1).

L'anomalie la plus élevée caractérisée par une géométrie subcirculaire couvre le dôme du Jebel Oust qui correspond à une structure anticlinale essentiellement crétacée à cœur liasique (Jauzein, 1957). Vers l'ouest, au niveau de la plaine à remplissage quaternaire située au nord du Jebel Mouine est identifiée une anomalie à forte amplitude qui peut être expliquée par l'effet d'un terrain dense plus profond. En effet, in situ, le forage d'eau 2 (Fig. 1b) montre sous une couche d'argiles épaisse de $16 \mathrm{~m}$, des alternances marno-calcaires du Barrémien. Elle peut être interprétée également comme une structure soulevée qui engendre généralement, à l'inverse d'une structure affaissée, une réponse positive (Prieto, 1996). Une anomalie d'ampleur comparable est localisée à proximité au niveau de l'affleurement crétacé du Jebel Oued El Hessi.

La mise en évidence des structures hautes qui comportent des dépôts calcaires perméables est un résultat très intéressant pour l'investigation des eaux souterraines dans la région de Bir M'Chergua.

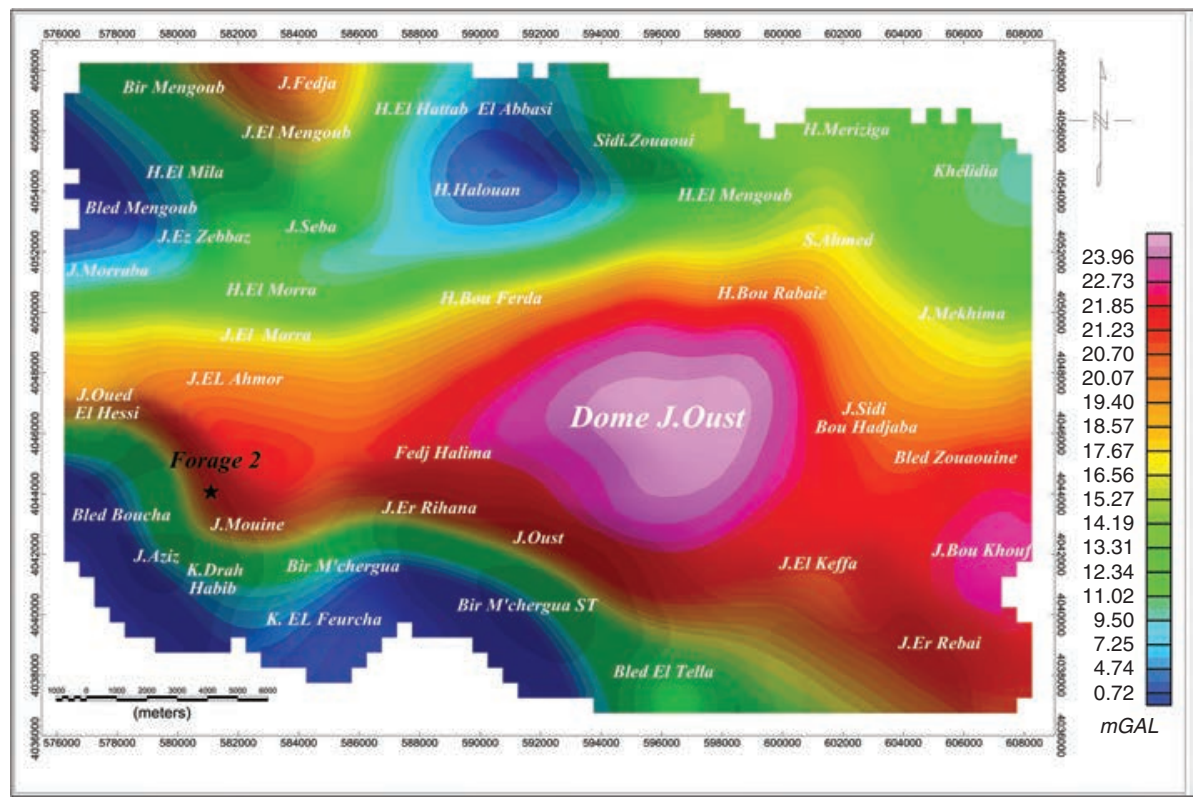

Fig. 5.-Carte d'anomalie du Bouguer de la région de Bir M'Chergua. 
Au nord -ouest du secteur d'étude, une anomalie à amplitude élevée caractérise Jebel Fedja qui correspond à un anticlinal constitué par les calcaires du Campanien, du Maestrichtien et de l'Eocène. A l'extrémité sud-est, Jebel Bou Khouf exprime aussi une forte réponse qui reflète sa structure anticlinale et sa composition en calcaires campaniens. Il s'agit d'un anticlinal secondaire du synclinal de Khélidia Ain El Djour qui occupe toute la partie orientale de la feuille de Bir M'Chergua (Fig. 1b). Cet anticlinal s'amortit rapidement vers le nord (Jauzein, 1957).

Les anomalies à faible amplitude couvrent la zone sud-ouest du secteur d'étude: la plaine quaternaire de Bir M'Cherga (Bled Smindja et plaine de Depienne) et la cuvette endoréique de Bled Boucha. Elles s'étendent aussi sur une grande partie de la zone septentrionale; au niveau des synclinaux de Bled el Mengoub (nord-ouest) et de Khelidia (nordest) et à Henchir el Mengoub (centre) qui fait partie de la plaine alluviale d'oued Chafrou.
La carte d'anomalie de Bouguer reflète l'expression de la majorité des structures en surface et celles qui sont enfouies. Toutefois elle révèle une réponse similaire pour Jebel Ez Zebbas et Henchir El Morra malgré que le premier soit un anticlinal et le second est un synclinal. Ils expriment une anomalie à moyenne amplitude (couleur verte). De surcroit, cette carte ne montre pas une anomalie spécifique à l'anticlinal jurassique du Jebel Aziz; sa réponse est influencée par celles des plaines quaternaires qui l'entourent.

\section{Cartes prolongées}

Pour une meilleure caractérisation des différentes structures géologiques proches de la surface, nous avons réalisé différents prolongements vers le haut (Fig. 6) afin de soustraire une composante régionale. Des modifications significatives sont enregistrées pour un prolongement de $3 \mathrm{~km}$ (Fig. 6a); les fortes anomalies au niveau du Jebel Fedja et la plaine

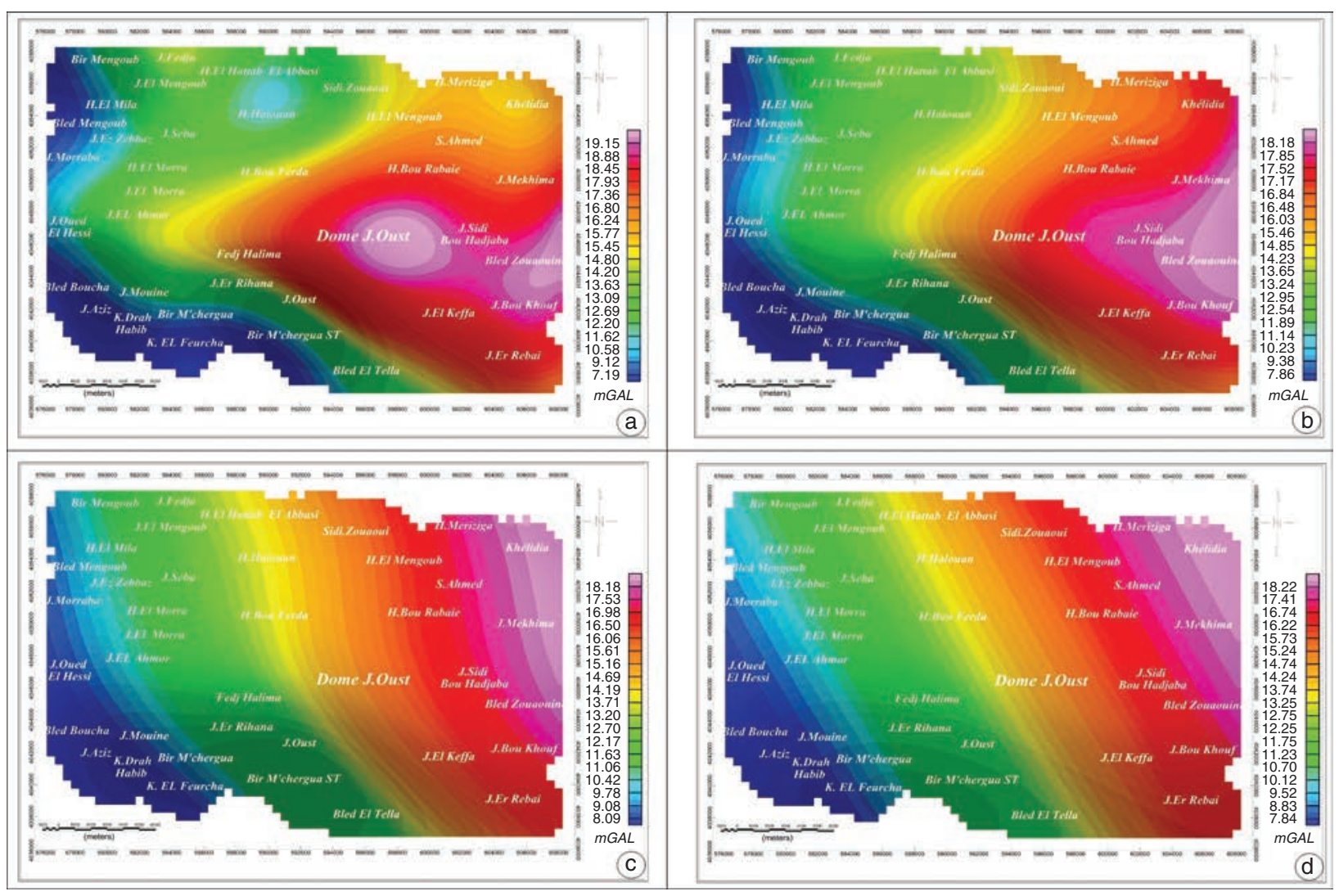

Fig. 6.-Prolongements vers le haut. 6 a. à une distance de $3 \mathrm{~km} .6 \mathrm{~b}$. à une distance de $5 \mathrm{~km}$. $6 \mathrm{c}$. à une distance de $10 \mathrm{~km}$. $6 \mathrm{~d}$. à une distance de $20 \mathrm{~km}$. 
quaternaire au nord du Jebel Mouine disparaissent indiquant le caractère peu profond des sources perturbatrices à l'origine de ces réponses gravimétriques. Aussi l'anomalie forte caractérisant le dôme du Jebel Oust commence à se fusionner avec celle de Jebel Bou Kouf. La carte prolongée à une distance de $5 \mathrm{~km}$ (Fig. 6b) montre l'installation d'un gradient gravimétrique croissant de l'ouest vers l'est. A un prolongement de $10 \mathrm{~km}$ (Fig. 6c), les courbes isogales sont caractérisées par un comportement lisse mais avec l'existence d'ondulations. A $20 \mathrm{~km}$ (Fig. 6d), ces ondulations sont complètement atténuées et la carte résultante représente la composante régionale $\mathrm{du}$ secteur d'étude. Elle révèle un gradient gravimétrique positif orienté SW-NE; un excès de masse au nordest et un déficit de masse au sud-est. Cette tendance peut être expliquée par l'amincissement de la croûte en allant du sud vers le nord de la Tunisie, mis en évidence à partir de la sismique profonde dans le cadre du programme de Geotraverse (Buness et al., 1992) et confirmé par la gravimétrie (Jallouli \& Mickus, 2000).

\section{Carte de l'anomalie résiduelle}

La soustraction de la composante régionale de l'anomalie de Bouguer a donné la carte résiduelle de la région de Bir M'Chergua (Fig. 7) qui montre des valeurs allant de -11à 12 mgal.
Comparée à la carte d'anomalie de Bouguer (Fig. 5), cette carte montre l'atténuation de l'anomalie positive du dôme du Jebel Oust qui peut s'expliquer par l'effet des dépôts alluvionnaires d'Oued Meliane. En revanche, elle révèle l'accentuation des anomalies positives situées au nord du Jebel Mouine, à Jebel Oued el Hessi et à Jebel Fedja. En outre, elle décèle des anomalies négatives bien définies à l'emplacement des dépressions quaternaires de Khélidia, Henchir el Mengoub qui reflètent la faible densité de leur remplissage. L'anomalie négative de la plaine de Bir M'Chergua (Bled Smindja et plaine de Depienne) et celle de la cuvette endoréique de Bled Boucha sont séparées par une anomalie positive à l'aplomb des affleurements crétacés supérieurs et éocènes de K. El Feurcha el kartine qui peut correspondre à une zone soulevée dont la ligne de crête constituerait une ligne de partage des eaux souterraines. Cette zone pourrait aussi jouer le rôle d'une barrière entre les eaux saumâtres de la Sabkha et les aquifères de la plaine de Bir M'Chergua.

L'anomalie négative de Bled El Mengoub s'atténue alors qu'à proximité à Jebel Ez Zebbas s'individualise une anomalie positive malgré la présence du Trias gypseux à faible densité à l'affleurement. Des anomalies positives caractérisant des structures salifères sont observées dans différentes localités de la Tunisie septentrionale telles que la haute vallée de Mejerda,

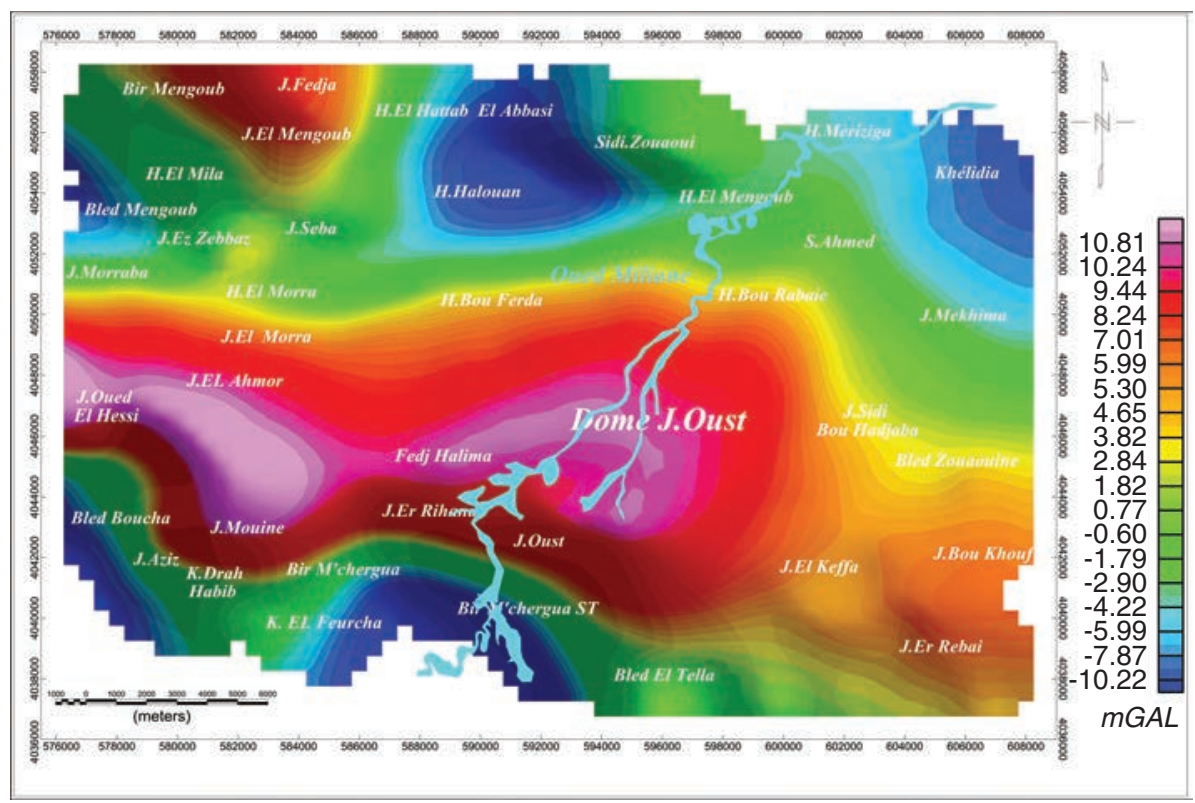

Fig. 7.-Carte d'anomalie résiduelle de la région de Bir M'Chergua. 
Jebel Cheid, Jebel Mourra et Lansarine (Benassi et al., 2006; Hamdi Nasr et al., 2009; Amiri et al., 2011; Sarsar Naouali et al., 2017). Ces réponses indiquent la présence des roches denses comme l'anhydrite et la dolomie en subsurface. Par ailleurs, des anomalies négatives sont les réponses d'autres corps salifères coexistants (Inoubli \& Mansouri, 2006).

\section{Carte de la dérivée verticale}

La carte de dérivée verticale de la région de Bir M'Chergua (Fig. 8) révèle l'individualisation et l'accentuation de certaines anomalies par rapport aux réponses enregistrées au niveau de la carte d'anomalie de Bouguer et même au niveau de la résiduelle. L'anomalie positive du Jebel Fedja s'étend plus au Sud indiquant la continuité de cette structure sous la couverture quaternaire. Celle du Jebel Ez Zebbas est remarquablement accentuée avec une forme subcirulaire et des limites bien définies. L'anomalie négative de Henchir el Mengoub s'allonge vers l'Ouest à Henchir el Morra et vers l'Est à Henchir Sidi El Hattab el Abbassi. A sa limite Nord, à Sidi Zouaoui, s'individualise une anomalie positive dirigée WNWESE qui peut constituer une structure haute composée de calcaires éocènes affleurant à proximité.

L'anomalie positive du dôme du Jebel Oust est bien matérialisée suivant une direction NE-SW surtout après sa séparation de l'anomalie du Jebel Bou Kouf. Ses amplitudes maximales sont vers la partie méridionale; elles couvrent l'affleurement crétacé inférieur du Jebel Er Rihana et le massif jurassique du Jebel Oust. Elle est faible dans la partie centrale du dôme où les affleurements sont essentiellement composés de dépôts quaternaires.

L'extension horizontale et l'orientation E-W de l'anomalie positive du Jebel Mouine sont mieux caractérisées malgré que sa réponse globale reste coalescente avec celle de l'anomalie du Jebel Oued el Hessi.

\section{Carte de la dérivée horizontale}

L'interprétation des maxima de la dérivée horizontale a permis d'identifier les discontinuités structurales majeures à l'échelle du secteur d'étude (Fig. 9a). Les alignements dégagés s'arrangent essentiellement suivant les directions E-W, NE-SW et NW-SE (Fig. 9b).

\section{Linéaments de direction NE-SW}

La direction NE-SW est représentée principalement par les linéaments F1, F2, F3, F4 et F5. F1 et F2 délimitent respectivement le dôme du Jebel Oust à l'est et à l'ouest. La technique de la déconvolution

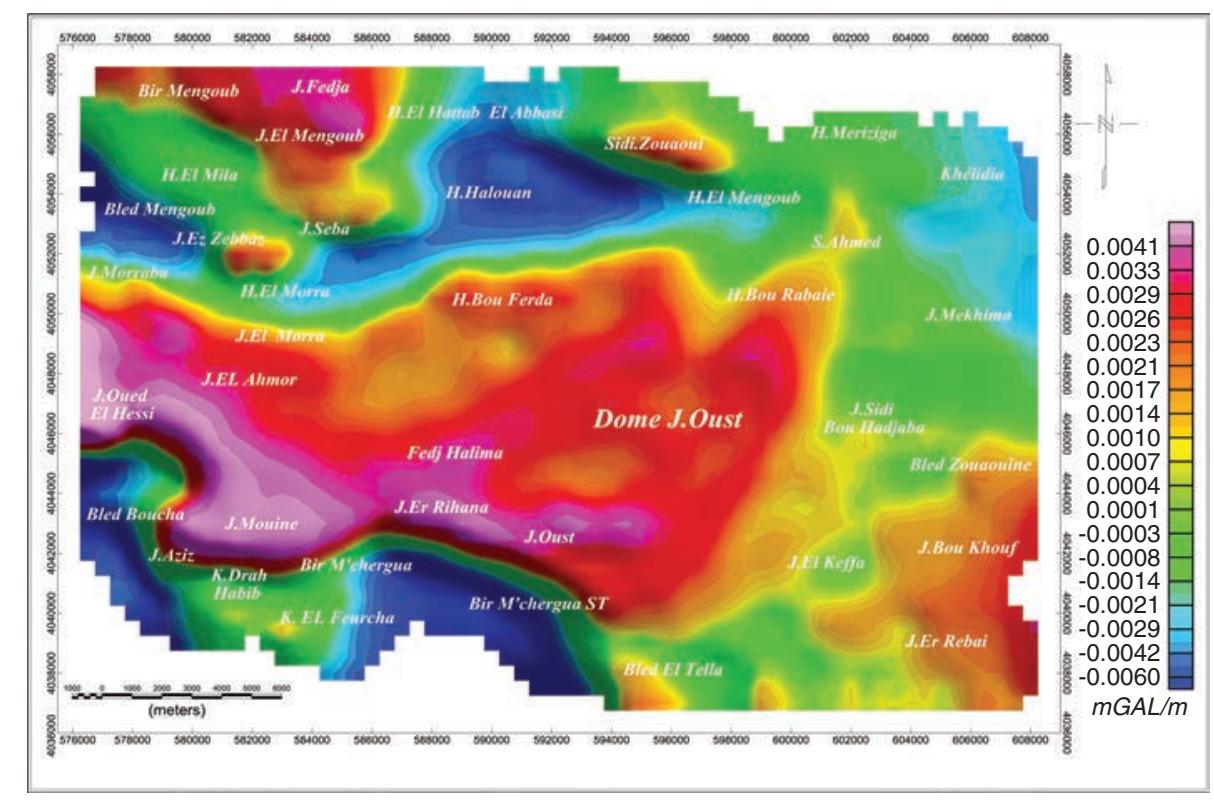

Fig. 8.-Carte de la dérivée verticale de la région de Bir M'Chergua. 


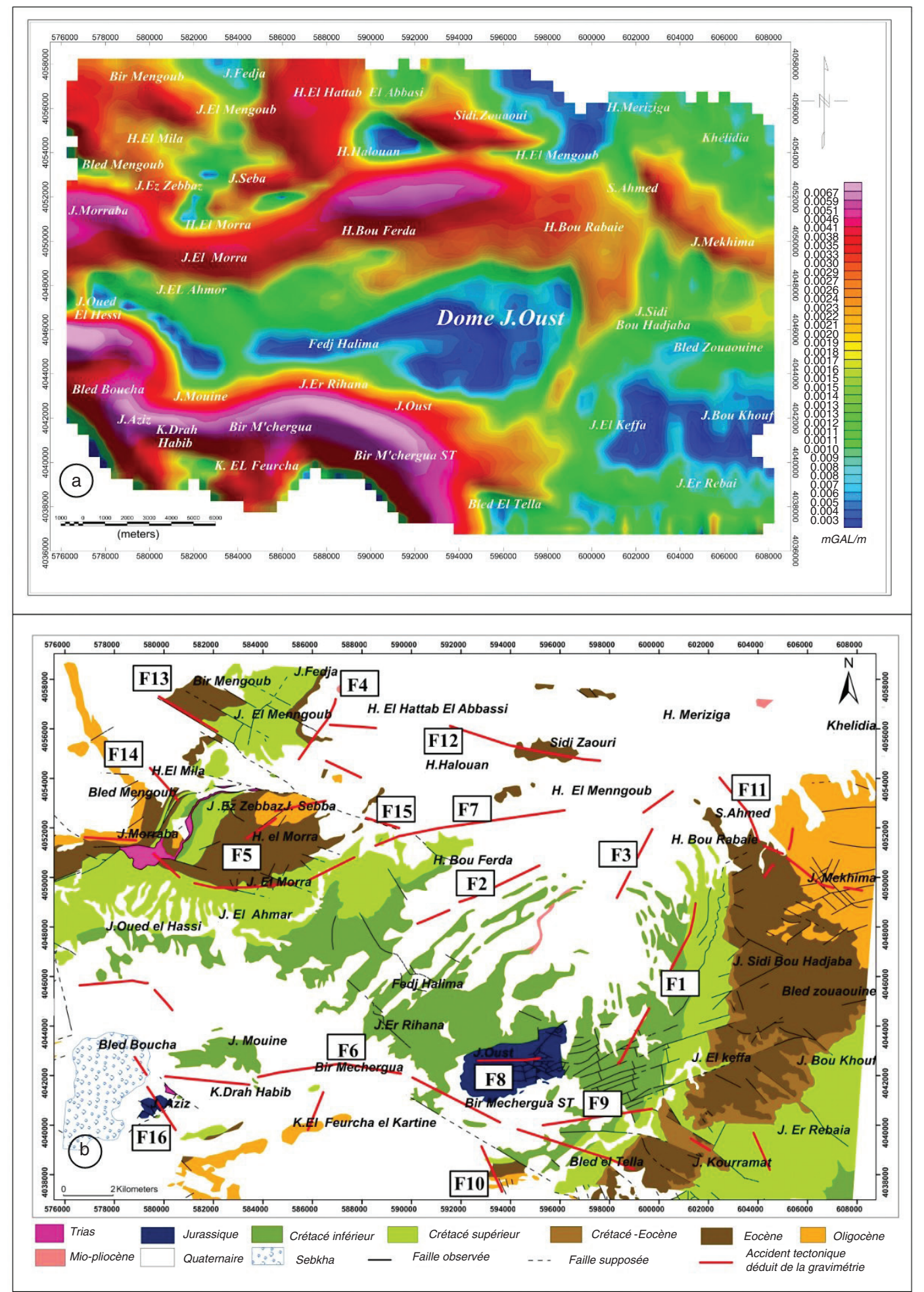

Fig. 9.-Linéaments déterminés de l'interprétation gravimétrique. 9a. Carte du gradient horizontal. 9b. Linéaments superposés à la carte géologique de Bir M'Chergua. 
d'Euler (Fig. 10) confirment les tracés de ces linéaments et décèlent leur caractère profond avec des solutions qui dépassent dans la majorité des points $1000 \mathrm{~m}$ pour $\mathrm{F} 1$ et $1500 \mathrm{~m}$ pour $\mathrm{F} 2$.

F3 qui tronque la partie septentrionale de ce dôme est aussi enraciné; sa profondeur estimée excède $1500 \mathrm{~m}$. L'alignement F4 borde à l'est la structure du Jebel Fedja alors que F5 tronque le synclinal de Henchir el Morra-Jebel Es Sba. Jebel Fedja est traversé par les failles NE-SW de Jerifet et el Fedja qui constituent des segments de l'accident régional de Tunis-Ellès à jeu actuel inverse senestre (Ben Chelbi et al., 2008). F4 et F5 peuvent être également interprétées comme des tronçons de cet accident.

Dans la partie méridionale de la carte, un alignement NE-SW peu étendu délimite à l'ouest la zone affaissée de Bir M'Chergua (Bled Smindja et plaine de Depienne).

Les linéaments NE-SW mis en évidence sont parallèles à la partie septentrionale de la faille plurikilométrique de Zaghouan qui prolonge la dorsale tunisienne (Rolland, 1885; Solignac, 1927; Castany, 1951; Turki, 1980; Creusot et al., 1992; Morgan et al., 1998) sur $80 \mathrm{~km}$ depuis Jebel Boukornine de Hammam Lif jusqu'à Jebel Bargou au Sud-Ouest. Cette faille correspond à un alignement composé de trois accidents distincts (Turki, 1980) qui sont du nord -est au sud-ouest: le chevauchement de Zaghouan proprement dit (partie septentrionale), l'accident subvertical du Jebel El Menassir-El Kemkine (partie centrale) et la faille d'effondrement du Jebel Serdj (partie méridionale).

\section{Linéaments de direction $E-W$}

La direction E-W est matérialisée essentiellement par quatre alignements: F6 », F7, F8 et F9. L'alignement majeur $\mathrm{F} 6$, d'importante extension horizontale, marque la limite entre les structures hautes de Jebels Oued El Hessi, Mouine, Er Rihane et les structures affaissées de Bir M'Chergua et Bled Boucha. Seul Castany (1951) a signalé le tracé de cet alignement. La déconvolution d'Euler (Fig. 10) révèle que la profondeur de F6 varie de 1000 à $1500 \mathrm{~m}$.

L'alignement F7 correspond à la limite méridionale des structures affaissées de Bled El Mengoub, Henchir el Mourra et Henchir el Mengoub et correspond à des profondeurs comprises essentiellement entre 1000 et $1500 \mathrm{~m}$.

Le troisième alignement F8 d'extension horizontale relativement faible affecte le massif jurassique du Jebel Oust. Ce massif est également orienté

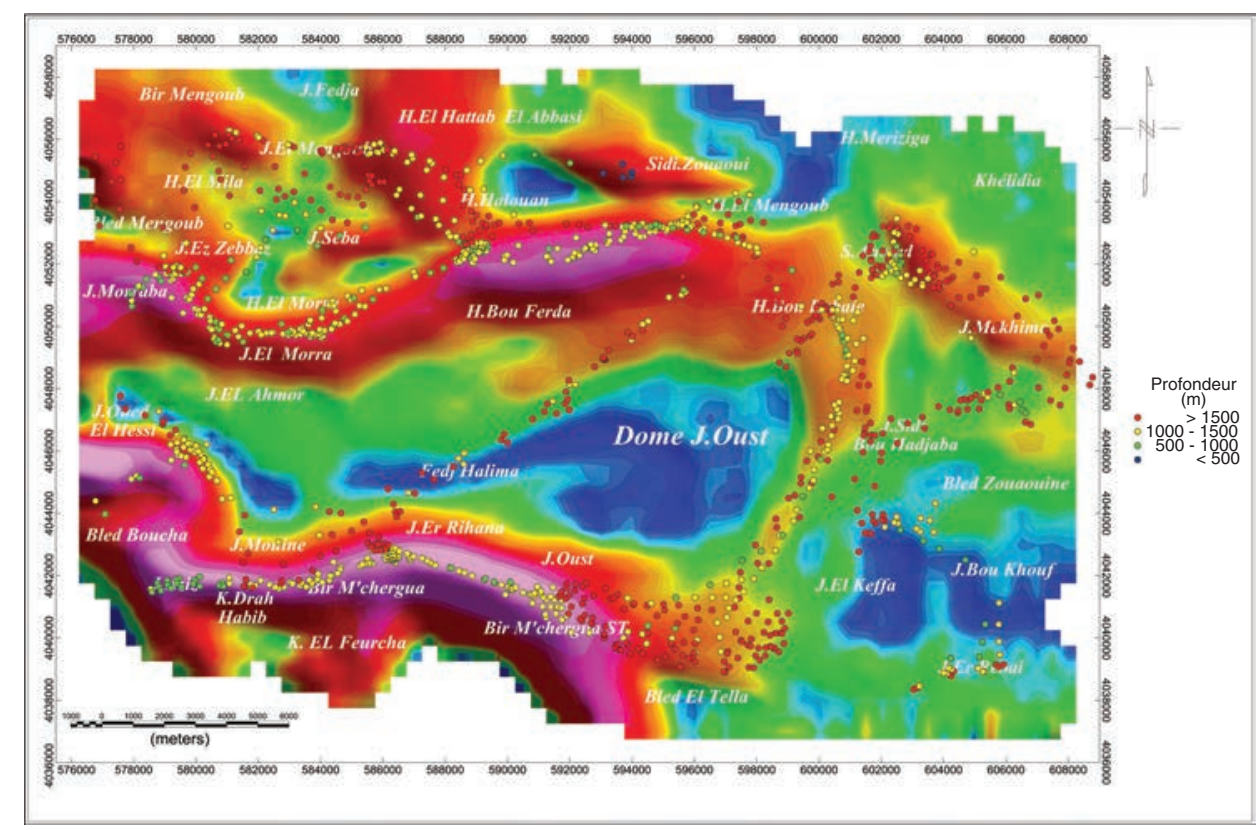

Fig. 10.-Solutions d'Euler pour l'indice structural IS $=0, F=8 \mathrm{~km}$ et $\mathrm{T}=20 \%$. 
E-W malgré qu'il constitue le cœur du dôme du Jebel Oust ayant une direction générale NE-SW (Jauzein, 1957).

Le quatrième alignement $\mathrm{F} 9$ est mis en évidence au niveau de l'affleurement crétacé couvrant la partie sud-est du dôme du Jebel Oust (Fig. 9b). Il est parallèle à plusieurs accidents identifiés en surface.

\section{Linéaments de direction NW-SE}

La direction NW-SE caractérise des alignements relativement nombreux mais d'extension latérale plus faible; il s'agit de F10, F11, F12 et F13 F14, F15 et F16.

L'alignement F10 coïncide en surface avec le réseau de failles transverses tronquant le bord sud du dôme du Jebel Oust (Fig. 9b) et délimitant au nord le fossé de Bir M'Chergua (Bajanik et al., 1977; Kadri \& Ben Haj Ali., 1999).

F11 ayant une géométrie en escalier se prolonge sur $5 \mathrm{~km}$ de Jebel Mekhma à Sidi Ahmed avec des profondeurs majoritairement supérieures à $1500 \mathrm{~m}$ (Fig. 10). Cet alignement marque la limite entre l'affleurement éocène du Jebel Bou Hadjaba et celui d'âge oligocène du Jebel Mekhma qui renferment des dépôts perméables composés respectivement de calcaires et de sables. Il peut constituer une faille butoir qui entrave l'écoulement entre les aquifères oligocène et éocène ou au contraire une zone de circulation préférentielle des eaux souterraines et favorise l'intercommunication de ces aquifères. F12 coïncide avec la limite méridionale de l'anomalie positive décelée à Sidi Zouaoui (Fig. 8). F13 coïncide avec l'accident déjà reconnu en surface qui délimite à l'ouest Jebel el Mengoub (Jauzein ,1957). «F14» borde au nord le synclinal de Bled el Mengoub -Henchir el Mila. F15 sépare les zones affaissées de Henchir El Mengoub et Henchir El Morra (Fig. 9b). La mise en évidence de cet alignement confirme en partie l'accident supposé sur la carte géologique qui s'étend du Jebel el Mengoub jusqu'à Henchir Bou Ferda (Fig. 9b). F16 marque la limite occidentale de l'anomalie négative couvrant la dépression de Bled Boucha (Fig. 8).

Dans la partie nord - ouest de la carte, sont mises en évidence des linéaments NW-SE d'extension relativement faible qui sont transversales à $\mathrm{F} 4$. Au sud-ouest, le linéament qui relie les segments de F6 limitant au sud Jebel Mouine et Jebel Oued Hessi explique la direction NW-SE de l'anomalie positive exprimée par la carte résiduelle (Fig. 7) et celle du gradient vertical (Fig. 8).

\section{Synthèse et conclusions}

L'interprétation qualitative et quantitative des anomalies gravimétriques a permis d'approcher au mieux l'organisation des structures profondes de la région de Bir M'Chergua qui sont généralement associées aux jeux de failles de différentes directions.

Les anomalies positives du dôme du Jebel Oust, Jebel Fedja et Jebel Mouine correspondent à des anticlinaux résultant de la compression NW-SE du Miocène supérieur qui correspond à la phase paroxysmale de l'édification et l'installation de l'Atlas tunisien (phase atlasique) (Ben Ayed \& Viguier, 1981; Bouaziz et al., 2002). Elles sont bordées par des accidents de direction NE-SW et E-W (Fig. 11). L'application de la compression N-S du Quaternaire ancien qui a donné la géométrie actuelle des structures atlasiques (Chihi, 1988; Mzali \& Zouari, 2006) dans notre zone d'étude accentue les plis et réactive les accidents NE-SW et $\mathrm{E}-\mathrm{W}$ en failles inverses avec une composante senestre pour les NE-SW.

Les anomalies négatives de Bir M'Chergua, Bled Boucha, Khelidia, Bled el Mengoub, Henchir el Mengoub et Henchir el Morra couvrent des dépressions d'accumulation de dépôts néogènes et quaternaires dont la plupart correspondent à des structures synclinales générées par la phase atlasique. Elles sont limitées par des linéaments orientés NE-SW, E-W et NW-SE (Fig. 11). Ces derniers répondent en failles normales à la compression N-S; les accidents tectoniques décrits en surface par Bajanik et al., 1977 et Kadri \& Ben Haj Ali, 1999 comme étant les limites du fossé de Bir M'Chergua -Mograne sont les meilleurs exemples. Seul le réseau des failles traçant la bordure nord- orientale de ce fossé est déterminé dans la feuille de Bir M'Chergua; il correspond à F10 mis en évidence à partir de l'interprétation gravimétrique.

Le fossé de Bir M'Chergua est cité parmi les grabens transversaux au tracé de l'accident de Zaghouan (Turki, 1988). 


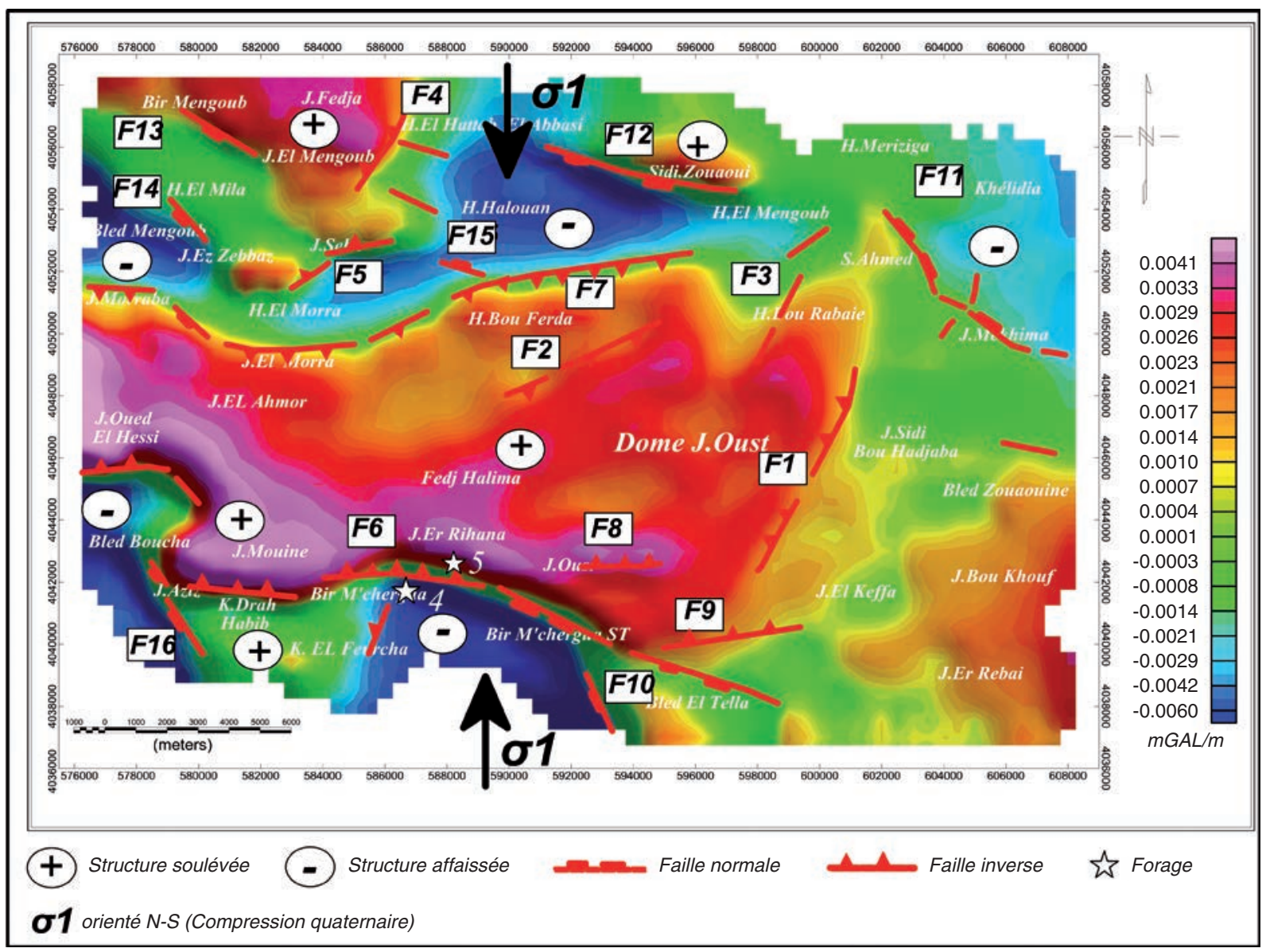

Fig. 11.-Schéma structural reconstitué de la région de Bir M'Chergua superposé à la carte de la dérivée verticale.

A l'extrémité Sud -Ouest de la feuille de Bir M'Chergua, la faille inverse F6 et la faille normale F16 ont contribué conjointement à l'affaissement de la cuvette synclinale de Bled Boucha. La zone haute entre cette cuvette et la plaine de Bir M'Chergua correspond à la fois au compartiment soulevé de F16 à l'ouest et d'une faille inverse à l'est.

L'intersection de deux accidents profonds F6 et F16 identifiés au niveau du Jebel Aziz facilite l'ascension du matériel triasique.

Dans la partie orientale, le synclinal de Khelidia - Ain Djour est affaissé d'avantage sous l'effet du jeu inverse de F1 qui le sépare du dôme du Jebel Oust. Par ailleurs le jeu normal de F11 a effondré la partie nord-est de ce synclinal, caractérisée par des amplitudes plus faibles que la partie sud-ouest.
Dans la partie septentrionale, la dépression de Henchir El Halouan- Henchir El Mengoub est un compartiment affaissé à la faveur de trois accidents tectoniques: les failles normales F12 et F15 respectivement au Nord et à l'Ouest et la faille inverse F7 au Sud. L'effet conjugué de ces déformations a engendré l'approfondissement de cette zone et a favorisé son remplissage par des dépôts quaternaires à faible densité et peut ainsi expliquer l'importante anomalie négative qui la couvre. Le compartiment soulevé de la faille F12 coïncide avec la zone haute mise en évidence au niveau de Sidi Zouaoui. Le synclinal de Henchir El Morra correspond au compartiment soulevé de la faille F15. Les accidents E-W à NE-SW à jeu inverse F5 et F7 séparent ce compartiment et les structures hautes au Nord-Ouest, au Nord et au Sud. 
L'anomalie négative de Henchir El HalouanHenchir El Mengoub est très accentuée par rapport à celle de Henchir El Morra. Ceci peut être expliqué par une profondeur plus importante et par l'existence des sédiments quaternaires.

A l'extrémité Nord-Ouest de la carte, le jeu normal des failles F13 et F14 a générée une succession d'effondrements, traduite par une diminution progressive des réponses gravimétriques, depuis l'anticlinal du Jebel Fedja jusqu'au synclinal du Bled El Mengoub dont la limite Sud est tracée par la terminaison occidentale de l'accident majeur F7.

Le schéma structural de Bir M'Chergua ainsi établi (Fig. 11) montre des traits tectoniques extensifs et d'autres compressifs non reconnus auparavant. La mise en évidence de ces traits permet une meilleure connaissance de la structuration de l'Atlas septentrional en Tunisie.

La coexistence de différents traits tectoniques dans la région étudiée offre une complexité à la géométrie du son système aquifère. Le plissement des séries sédimentaires, la réactivation des accidents NE-SW et E-W qui délimitent les bombements anticlinaux de cuvettes synclinales en failles inverses et le jeu normal des accidents NW-SE sont à l'origine de la compartimentation de ce système en structures soulevées et affaissées. Les structures soulevées peuvent constituer des zones de partage des eaux ainsi que des zones de recharge préférentielles des dépôts perméables particulièrement au niveau des affleurements de calcaires crétacés qui constituent les principaux réservoirs dans ces zones. Les zones affaissées renferment généralement un aquifère multicouche; les couches crétacées atteintes à une profondeur relativement importante sont surmontées par d'autres tertiaires et/ou quaternaires.

Les structures reconstituées peuvent ainsi expliquer le changement de la profondeur d'une formation perméable entre deux points d'eau voisins tel est le cas des forages 4 et 5 (Fig. 11). En effet, l'aquifère barrémien est recoupé uniquement à $11 \mathrm{~m}$ de profondeur par le forage 5 situé au niveau du compartiment soulevé de la faille inverse F6 alors qu'il est atteint à $40 \mathrm{~m}$ au niveau du forage 5 implanté dans le compartiment affaissé de cette faille (Fig. 12). Cette faille est enracinée et peut aussi générée des variations de profondeur ainsi que d'épaisseur dans les séries sédimentaires plus profondes, non atteintes par les forages d'eau 4 et 5 .

Ces structures peuvent aussi jouer un rôle dans les relations entre les différents aquifères caractérisant la région et influencer la circulation et les caractéristiques des eaux souterraines. Les eaux thermales du Jebel Oust constituent un très bon exemple.

En effet, un modèle gravimétrique 2.5D de direction NNW-SSE qui recoupe le dôme du Jebel Oust (Fig. 13) a permis de déceler d'avantage la géométrie de cette structure et son effet sur l'écoulement souterrain.

La modélisation a été réalisée en utilisant le logiciel GM-SYS (Geosoft), les contrastes de densité ont été calculés par rapport à la densité de réduction des mesures gravimétriques. Les géométries des couches ont été construites en intégrant les différentes coupes géologiques construites par Solignac, 1927 et Castany, 1951 et en adoptant des densités moyennes représentatives des formations géologiques de la zone d'étude (Table 2).

Le modèle gravimétrique a été ajusté en comparant la réponse gravimétrique calculée à celle extraite de la carte résiduelle calculée d'un prolongement de $5 \mathrm{~km}$ (Fig. 13a).

Le modèle obtenu (Fig. 13b) révèle une concordance avec les résultats de l'interprétation qualitative; la forte anomalie coïncide avec la présence de calcaires jurassiques et crétacés. La faible anomalie correspond à la réponse des dépôts quaternaires de faible densité.

Les séries jurassique et crétacée du dôme du Jebel Oust sont affectées par des accidents tectoniques d'importante profondeur qui dépasse par endroits $1500 \mathrm{~m}$. cette structuration explique l'origine des eaux thermales à forte salinité captées par le forage 7, implanté au niveau du Jebel Oust (massif jurassique); les accidents mis en évidence favorisent une pénétration profonde des eaux de recharge ainsi que leur contact avec les terrains triasiques riches en sels.

La présente étude a abouti à des résultats qui peuvent contribuer énormément à une exploitation rationnelle des ressources en eau souterraine de la région de Bir M'Chergua. L'intégration d'autres méthodes géophysiques telle que la sismique 


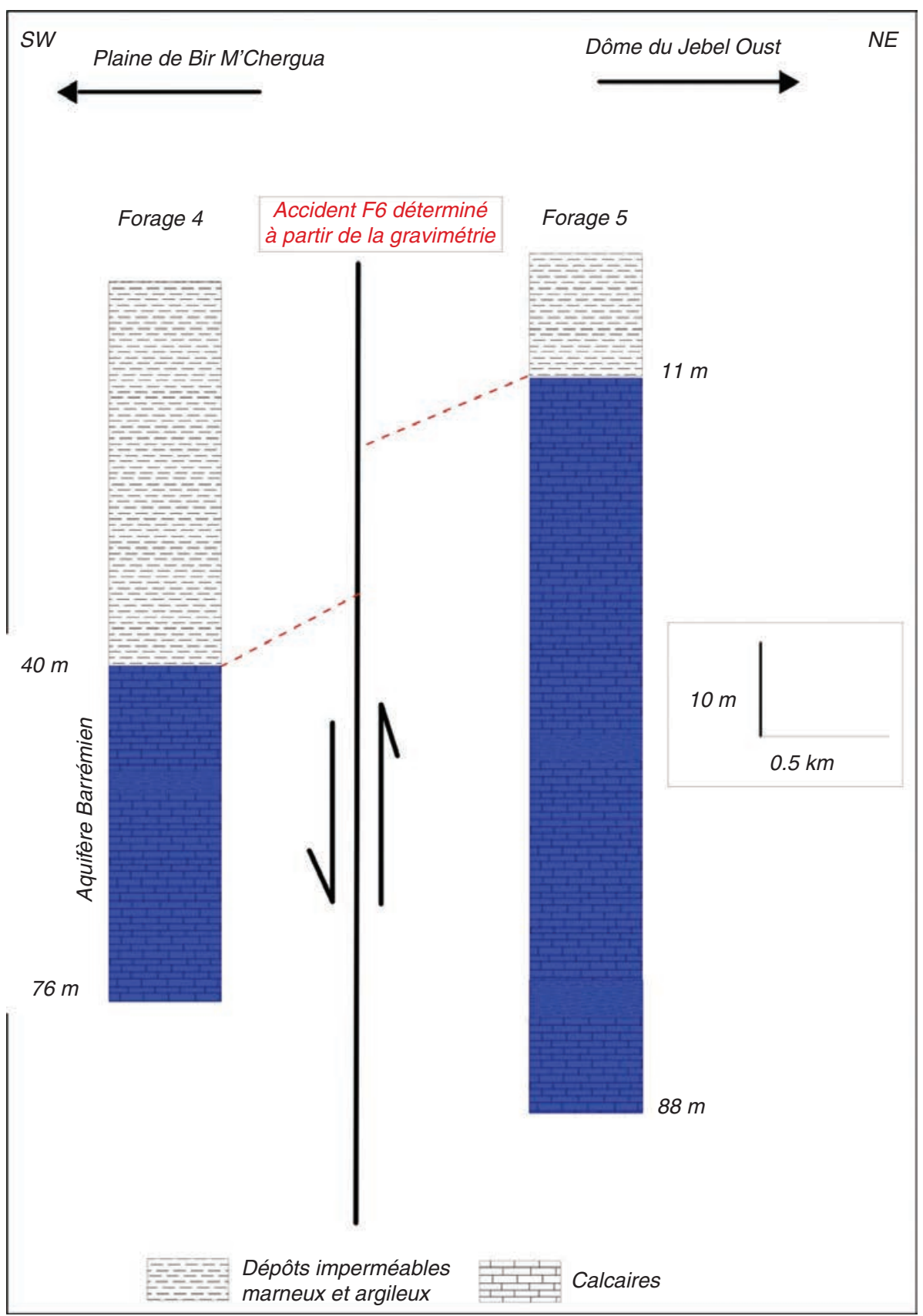

Fig. 12.- Variation de la profondeur de l'aquifère barrémien sous l'effet de l'accident tectonique F6.

réflexion qui est actuellement inaccessible dans cette région ne peut qu' améliorer ces résultats et apporter plus des précisions au schéma structural établi. Par ailleurs cette étude peut servir d'exemple pour d'autres projets ayant pour objectif une meilleure gestion des eaux dans les régions arides dans le monde.

\section{REMERCIEMENTS}

Les auteurs remercient Mr. Farhat Ben M'Barek, Directeur du Service Géophysique à l'Office National des Mines, de leur avoir fourni les mesures gravimétriques. Ils remercient également les deux reviewers anonymes pour leurs remarques constructives. 


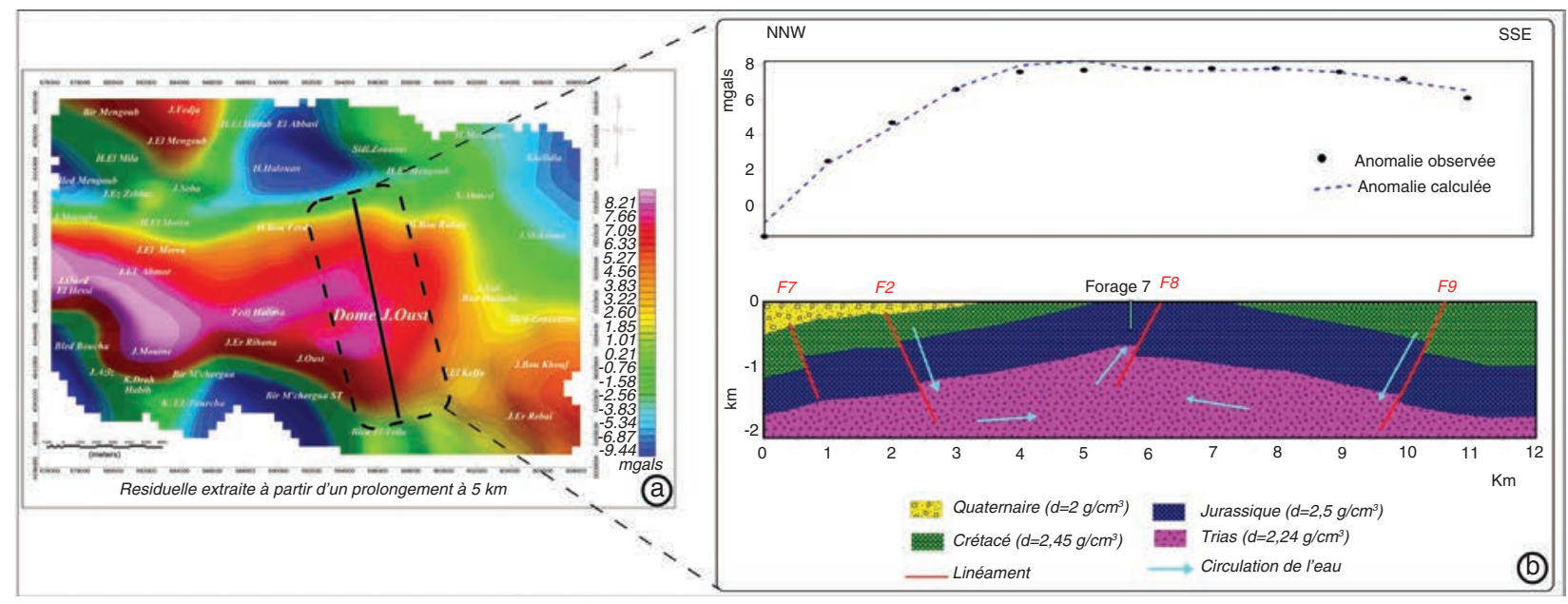

Fig. 13.-Modélisation gravimétrique 2.5D au niveau du dôme du Jebel Oust.

Table 2.-Densités moyennes représentatives des formations géologiques de la zone d'étude.

\begin{tabular}{llc}
\hline Âge & Lithologie & Densité $\left(\mathrm{g} / \mathrm{cm}^{3}\right)$ \\
\hline Trias & Gypse, grès, argile avec des minces lits de calcaires et de dolomies & 2,24 \\
Jurassique & Calcaires avec intercalations marneuses & 2,5 \\
Crétacé & Alternances marno-calcaires & 2,45 \\
Quaternaire & Sables, argiles, galets, graviers et calcaires & 2 \\
\hline
\end{tabular}

\section{Références}

Amiri, A.; Chaqui, A.; Hamdi Nasr, I.; Inoubli, M.H.; Ben Ayed, N. \& Tlig, S. (2011). Role of preexisting faults in the geodynamic evolution of northern Tunisia, insights from gravity data from the Medjerda valley. Tectonophysics, 506: 1-11. https://doi.org/10.1016/j. tecto.2011.03.004

Bajanik, S.; Biely, A.; Mencik, E.; Salaj, J. \& Stranik, Z. (1977). Notice explicative de la carte géologique 1/50.000 de Zaghouan. Service géologique de la Tunisie.

Benassi, R.; Jallouli, C.; Hammami, M. \& Turki, M.M. (2006). The structure of Jebel El Mourra, Tunisia: a diapiric structure causing a positive gravity anomaly. Terra Nova, 18: 432-439. https://doi. org/10.1111/j.1365-3121.2006.00709.x

Ben Ayed, N. \& Viguier, C. (1981). Interprétation structurale de la Tunisie atlasique. Comptes Rendus de l'Académie des Sciences, 292: 5-20.

Ben Chelbi, M.; Melki, F. \& Zargouni, F. (2008). Précision sur l'évolution structurale de l'Atlas 467 septentrional de Tunisie depuis le Crétacé (Bassin de Bir M'Cherga). Echos d'une 468 évolution polyphasée de la marge tunisienne dans son cadre méditerranéen. Africa Geosciences Review, 3: 229-246.
Blakely, R.J. (1996). Potential Theory in Gravity and Magnetic Applications. Cambridge University Press, $461 \mathrm{pp}$.

Bouaziz, S.; Barrier, E.; Soussi, M.; Turki, M.M. \& Zouari, H. (2002). Tectonic evolution of the northern African margin in Tunisia from paleostress data and sedimentary record. Tectonophysics, 337: 227-253. https://doi.org/10.1016/S0040-1951(02)00370-0

Buness, H.; Giese, P.; Bobier, C. ; Eva, C.; Merlanti, F.; Pedone, R.; Jenatton, L.; Nguyen, D.T.; Thouvenot, F.; Egloff, F.; Makris, J.; Lozej, A.; Maistrello, M.; Scarascia, S.; Tabacco, I.; Burollet, P.F.; Morelli, C.; Nicolich, R.; Zaghouani, T.; Egger, A.; Freeman, R. \& Mueller, S. (1992). The EGT'85 seismic experiment in Tunisia. A reconnaissance of the deep structures. Tectonophysics, 207: 245-267. https://doi. org/10.1016/0040-1951(92)90479-P

Castany, G. (1951). Etude géologique de l'Atlas tunisien oriental. Thèse es-Sciences France. Annales de Mines et de la Géologie n8, Tunisie, 632 pp.

Chihi, L. (1988). Déformations tectoniques quaternaires en Tunisie centrale (région de Kasserine). Géologie Méditerranéenne, 3: 177-182.

Dubois, J.; Diament, M. \& Cogne, J.P. (2011). Géophysique. Cours et exercices corrigées, 4ème édition. Dunod, Paris, 257 pp. 
El Goumi, N.; Jaffal, M.; Kchikach, A. \& Manar, A. (2010). Apport de la gravimétrie à l'étude de la structure du bassin du Haouz (Maroc). Estudios Geologicos, 66: 181-191. https://doi.org/10.3989/egeol.40051.082

Fedi, M. \& Florio, G. (2001). Detection of potential fields source boundaries by enhanced horizontal derivative method. Geophysical prospecting, 49: 40-58. https:// doi.org/10.1046/j.1365-2478.2001.00235.x

Gabalda, G.; Bonvalota, B.S. \& Hipkin, R. (2003). CG3TOOL; an interactive computer program to process Scintrex CG-3/3M gravity data for high-resolution applications. Computers \& Geosciences, 29: 155-171. https://doi.org/10.1016/S0098-3004(02)00114-0

Hamdi Nasr, I.; Inoubli, M.H.; Ben salem, A.; Tlig, S. \& Mansouri, A. (2009). Gravity contributions to the understanding of salt tectonics from the Jebel Cheid area (dome zone, Northern Tunisia). Geophysical Prospecting, 57: 719-728. https://doi. org/10.1111/j.1365-2478.2009.00788.x

Inoubli, M.H. \& Mansouri, A. (2006). Apport de la gravimétrie dans la caractérisation des modèles de mise en place des structures triasiques à caractère extrusif. 21ème Colloque Africain de Géologie, Maputo, Mozambique.

Jacobsen, B.H. (1987). A case for upward continuation as a standard separation filter for potential field maps. Geophysics, 52: 1138-1148. https://doi. org/10.1190/1.1442378

Jacoby, W. \& Smilde, P. L. (2009). Gravity Interpretation, Fundamentals and Application of Gravity Inversion and Geological Interpretation. Springer, Heidelberg, 395 pp.

Jallouli, C. \& Mickus, K. (2000). Regional gravity analysis of the crustal structure of Tunisia. Journal of African Earth Sciences, 30: 63-78 https://doi.org/10.1016/ S0899-5362(00)00008-7

Jauzein, A. (1957). Carte géologique de Bir M'Cherga à l'échelle 1:50000. Service Géologique de Tunisie.

Jauzein, A. (1957). Notice explicative de la carte géologique de Bir M'Cherga à l'échelle 1:50000. Service Géologique de Tunisie.

Kadri, A. \& Ben Haj Ali, M. (1999). Eléments de réflexion sur les linéaments tectoniques Est-Ouest et NordSud et les grabens associés en Tunisie septentrionale: Notes du service géologique de Tunisie, 65: 131-140.

Morgan, M.; Grocott, J. \& Moody, R. (1998). The structural evolution of the Zaghouan-Ressas Structural Belt, northern Tunisia. Geological Society London, 132: 405-422. https://doi.org/10.1144/GSL. SP.1998.132.01.23

Mzali, H. \& Zouari, H. (2006). Caractérisation géométrique et cinématique des structures liées aux phases compressives de l'Éocène au Quaternaire inférieur en Tunisie: exemple de la Tunisie nord-orientale. Comptes Rendus de l'Académie des Sciences, 338: 742-749 https://doi.org/10.1016/j.crte.2006.05.003
Najine, A.; Jaffal, M.; El Khammari, K.; Aïfa, T.; Khattach, D.; Himi, M.; Casas, A.; Badrane, S. \& Aqil, H. (2006). Contribution de la gravimétrie à l'étude de la structure du bassin de Tadla (Maroc): Implications hydrogéologiques. Comptes Rendus de l'Académie des Sciences, 338: 676-682. https://doi. org/10.1016/j.crte.2006.04.015

Nely, G. (1986): Les séries à évaporites en exploration pétrolière. 2 méthodes géophysiques. Technip, Paris, $258 \mathrm{pp}$.

Ouerghi, S.; Rebai, N.; Gabtni, H.; Farhat, F. \& Bouaziz, S. (2013). Apport de la gravimétrie à l'étude des structures effondrées du Nord-Est de la Tunisie: implications hydrogéologiques. Hydrological Sciences Journal, 58: 1-13. https://doi.org/10.1080/026 26667.2013.818219

Prieto, C. (1996). Gravity/Magnetic signatures of variations geologic models exercise in parttern recognition. Integrated Geophysics Corporation. Footnote series, 4: 1-26.

Reid, A.B.; FitzGerald, D. \& McInerney, P. (2003). Euler deconvolution of gravity data. 73rd Annual International Meeting, SEG, Expanded Abstracts, 576-579. https://doi.org/10.1190/1.1817993

Rolland, G. (1885). Sur la montagne et la grande faille de Zaghouan (Tunisie). Comptes Rendus de l'Académie des Sciences, 101: 1187-1190.

Sarsar Naouali, B.; Guellala, R.; Bey, S. \& Inoubli, M.H. (2017). Gravity Data Contribution for Petroleum Exploration Domain: Mateur Case Study (Saliferous Province, Northern Tunisia). Arabian Journal for Science and Engineering, 42: 339-352. https://doi. org/10.1007/s13369-016-2152-0

Schoeffler, J. (1975). Gravimétrie appliquée aux recherches structurales et à la prospection pétrolière et minière. Technip, Paris, 288 pp.

Solignac, M. (1927). Etude géologique de la Tunisie septentrionale. Thèse d'Etat, $756 \mathrm{pp}$.

Thompson, D.T. (1982). EULDPH: a new technique for making computer-assisted depth estimates from magnetic data. Geophysics, 47: 31-37. https://oi. org/10.1190/1.1441278

Turki, M.M. (1980). La “faille de Zaghouan” est la résultante de structures superposées (Atlas Tunisien Central). Bulletin de la Société Géologique de France, 3: 321-325. https://doi.org/10.2113/gssgfbull. S7-XXII.3.321

Turki, M.M. (1988). Polycinématique et contrôle sédimentaire associé sur la cicatrice Zaghouan-Nebhana. Thèse d'Etat, Université de Tunis, 252 pp.

Zeng, H.; Xu, D. \& Tan, H. (2007). A model study for estimating optimum upward continuation height for gravity separation with application to a Bouguer gravity anomaly over a mineral deposit, Jilin province, northeast China. Geophysics, 72: 145-150. https://doi.org/10.1190/1.2719497 